\title{
Progetto di uno spettroeliografo per Lyman-Alfa con telescopio per satelliti $(*)$
}

\author{
F. Potenzi
}

Ricevuto il 19 Febbraio 1963

\begin{abstract}
Riassunto. - Viene descritto un sistema per effettuare la scansione dell'immagine solare nel campo di lunghezze d'onda della Lyman-Alfa. Esso consiste in un'ottica a specchi tipo Cassegrain, un sistema di orientazione, uno spettroeliografo per la dissezione dell'immagine solare e $\mathrm{nn}$ sistema di codificazione e trasmissione. Vengono descritte alcune prove e controlli da eseguire prima del lancio.
\end{abstract}

SUMMARY. - A satellite-borne system for dissecting the solar image in the Lyman-Alfa wavelength range is outlined. It consist of a Cassegrain mirror optics, an orienting device, a spectroheliograph dissecting the solar image and a coding and transmitting system. Some tests to perform before launching are described.

Il sistema rappresentato nella figura è uno spettroeliografo per la Lyman alfa con telescopio per raccogliere l'immagine solare e sistema di stabilizzazione. L'immagine solare viene costruita da un sistema Cassegrain. Esso è il sistema più leggero e più luminoso (anche col sole, a queste lunghezze d'onda la luminosità è importante) che permette agevolmente di ottenere una lunga focale (ad esempio 2 metri), con un ingombro molto limitato. Esso è sostanzialmente un teleobbiettivo a specchi. A $1250 \mathrm{~A}$ il potere riflettente dell'alluminio e del rodio è basso ma sempre abbastanza elevato (ca. $40-50 \%$ ) per il sole.

Questo telescopio è orientato verso il sole mediante la piattaforma stabilizzata della Società Norden (di cui si vede il profilo in basso a sinistra), essa pesa soltanto 20 libbre ed è costituita da un sistema giroscopico

$\left(^{*}\right)$ Nota presentata al $2^{\circ}$ Congresso Internazionale Tecnico Scientifico dello Spazio. Roma, 19-23 Giugno 1962. 
il quale permette di riprodurre mediante una coppia Selsyn l'orientazione che devono possedere $i$ due assi di una montatura azimutale del telescopio allo scopo di mantenere la sua orientazione nello spazio costante entro pochi secondi d'arco, e indipendentemente dalla orientazione nello spazio della intelaiatura di supporto (incolucro del satellite). Tale piattaforma stabilizzata è stata già estensivamente utilizzata su satelliti per scopi analoghi e fortunatamente la sua utilizzazione non è più oggi un problema.

Il disegno mostra l'uscita dei segnali elettrici per il pilotaggio dei due assi del telescopio (filo rosso) tramite i Selsyn (i cilindri beige sughi assi ortogonali).

La piattaforma stabilizzata va orientata in modo che il telescopio punti il sole nel modo desiderato prima del lameio dell'apprarecehiatura; essa poi manterrà la sua orientazione indipendentemente dalle vicissitudini impostele dal vettore.

L'immagine solare viene proiettata sulla fenditura di un monocromatore ad alta dispersione che utilizzerà quindi come mezzo dispersivo, date anche le caratteristiche della radiazione da osservare, un reticolo concavo; evitando quindi l'impiego di altra ottica.

Sulla fenditura di uscita è piazzato il dispositivo di movimento delle fenditure per la scansione dell'immagine solare (esso non è mostrato nel disegno).

Nel caso in cui si possa effettuare il ricupero del satellite (cosa assai improbabile) l'immagine potrà essere costruita su di una emulsione fotografica, altrimenti verrà inviato a un fotometro che, man mano che l'immagine si sposta di fronte alla fenditura, raccoglie il diagramma delle intensità lungo la fenditura e lo invia al sistema di trasmissione dati a terra, indicato nell'angolo inferiore destro del disegno.

Lo schizzo non mostra i blocchi del sistema di ricezione e codificazione dei dati presi dalla fenditura; esso non mostra altresì i sistemi che, internamente allo spettrografo, eliminano l'arrivo, sulla fenditura di uscita, di luce di lunghezza d'onda non desiderata.

Il fotomoltiplicatore per il rilievo dei dati sulla fenditura esige, per il suo funzionamento, che la radiazione della Lyman alfa sia trasformata, per esempio mediante un composto fluorescente, in una radiazione per cui è trasparente la chiusura del fotomoltiplicatore dalla parte del fotoratodo (quarzo), oppure, dato che il sistema funzionerà nel vuoto spinto il fotomoltiplicatore potrà addirittura essere privo della finestra di chiusur"l.

Un programma minimo potrà consistere nel rilevamento della sola intensità della Lyman alfa emessa da una particolare regione del sole; 
i dati relativi verranno trasmessi continuamente a terra per essere correlati ad altre manifestazioni dell'attività solare rilevabili anche a terra.

Il programma di studio per la messa a punto di siffatta apparechiatura comprende:

1 - La scelta delle dimensioni del telescopio e la sua costruzione, messa a punto in laboratorio. Si prenderanno in considerazione materiali extraleggeri (alluminio e magnesio per le strutture, berillio per lo specchio, etc.).

2 - Costruzione, messa a punto e collaudo del sistema di controllo del puntamento; anche questa costruzione è realizzata con i materiali più leggeri, dovrà venire collaudata in laboratorio, su di una piattaforma che il collaudatore farà orientare a capriccio, o vibrare controllando l'efficacia del sistema di puntamento ad annullare questi falsi movimenti tenendo in puntamento l'immagine solare. Questa è la chiave per ottenere una buona qualità della immagine.

3 - Costruzione e collaudo del prototipo del sistema di rilevamento e codificazione dell'immagine da teletrasmettere; in particolare si dovrà decidere se rilevare sulla fenditura di uscita le intensità con un fotumetro di tipo classico o con un contatore di singoli fotoni. Costruito il prototipo, si dovrà costruire una prima rersione della struttura definitiva, miniaturizzata ed alleggerita da provare per la sua efficacia di funzionamento, insieme al sistema 2, in una camera a vuoto spinto, soprattutto per controllare la resistenza delle parti elettriche.

4 - Infine sono opzionali e consigliabili prove generali di funzionamento del sistema (eventualmente non su Lyman alfa), per esempio con lanci in pallone con ricupero. Prove di questo genere sono estremamente importanti per la sicurezza del funzionamento finale. 